\begin{tabular}{|c|c|c|}
\hline $\mathrm{MS}^{\mathrm{M}}$ & $\begin{array}{l}\text { Journal of Mathematical Sciences and Modelling, 3 (3) (2020) 139-143 } \\
\text { Research Article } \\
\text { Journal of Mathematical Sciences and Modelling } \\
\text { Journal Homepage: } \text { www.dergipark.gov.tr/jmsm } \\
\text { ISSN } 2636-8692 \\
\text { DOI: } \text { http://dx.doi.org/10.33187/jmsm.810655 }\end{array}$ & $\begin{array}{l}\text { JOURNAL OF } \\
\text { MAIHAMATICAL } \\
\text { SCIENCES AND } \\
\text { MODELIING } \\
-\end{array}$ \\
\hline
\end{tabular}

\title{
On Bicomplex Jacobsthal-Lucas Numbers
}

\author{
Serpil Halici ${ }^{1}$ \\ ${ }^{1}$ Department of Mathematics, Faculty of Science and Arts, Pamukkale University, Denizli, Turkey
}

\section{Article Info}

Keywords: Bicomplex numbers, Jacobsthal sequence, Recurrences.

2010 AMS: 11B37, 11B39, 11R52.

Received: 14 October 2020

Accepted: 28 December 2020

Available online: 29 December 2020

\begin{abstract}
In this study we introduced a sequence of bicomplex numbers whose coefficients are chosen from the sequence of Jacobsthal-Lucas numbers. We also present some identities about the known some fundamental identities such as the Cassini's, Catalan's and Vajda's identities.
\end{abstract}

\section{Introduction and preliminaries}

Quaternionic numbers, defined by Hamilton in 1843 , led to the existence of an algebraic structure with all the properties of real and complex numbers, except the property of change of multiplication. There are also many studies on quaternions' coefficients( see, [1],[2],[3]). The recognition and identification of bicomplex numbers was made by James Cockle[4]. Cockle defined a bicomplex number as $b=z_{1}+\mathbf{j} z_{2}$ using the new unit $\mathbf{j}$, which Hamilton described, inspired by the definition of quaternions. In 1892, Segre gave different interpretations of this algebra by studying again on bicomplex numbers algebra[5]. In [6], Price, while dealing with the field properties of bicomplex numbers, some authors [7], [8], [9],[10] made some important studies on bicomplex holomorphic functions. In the last years, some studies have been done on bicomplex numbers. In particular, one can refer to the related sources for some studies made using different representations of these numbers [11], [9]. Furthermore, there are some studies on bicomplex numbers and their algebraic, geometric, topological and dynamic properties. For some of these studies, the reader can look at [12], [13], [4],[14], [15]. Recently, the studies conducted by selecting the coefficients of bicomplex numbers from different sequences have attracted attention. Because it is easier to work with these new sequences created using the properties of the selected a sequence, it is also easier to find application areas in other fields. One of these studies, the coefficients by selecting from the Fibonacci sequence and using idempotent notation, by made Halici[11]. Jacobsthal and Jacobsthal-Lucas sequences have a rich history, especially in view of its relationship to the Fibonacci numbers these are studied by some authors. Cerin, in [13], examined the products of Jacobsthal numbers and gave sum of their squares. A. F. Horadam studied the Jacobsthal representation numbers and Fibonacci quaternions [16],[17]. Szynal-Liana and Włoch worked on Jacobsthal quaternions[18]. Diana, in [19], considered Fibonacci octonions and generalized Fibonacci-Lucas octonions. Jacobsthal and Jacobsthal-Lucas sequences, respectively, are defined by the aid of the following recursive relations[20]: For $n \geq 2$

$$
J_{n}=J_{n-1}+2 J_{n-2} ; \quad J_{0}=0, \quad J_{1}=1
$$

and

$$
j_{n}=j_{n-1}+2 j_{n-2} ; \quad j_{0}=2, \quad j_{1}=1 .
$$

Moreover, these sequences can be also given by the following formulas: For $n \geq 0$

$$
J_{n}=\frac{1}{3}\left\{2^{n}-(-1)^{n}\right\}, \quad j_{n}=2^{n}+(-1)^{n} .
$$

We listed some important identities used some of them:

$$
\sum_{i=1}^{\infty} j_{i} x^{i-1}=(1+4 x)\left(1-x-2 x^{2}\right)^{-1}
$$




$$
\begin{gathered}
j_{n+1}+j_{n}=3 \cdot 2^{n}, n \geq 0 . \\
j_{n+1}-j_{n}=2^{n}-2(-1)^{n}, n \geq 0 . \\
j_{n+r}+j_{n-r}=2^{n-r}\left(2^{2 r}+1\right)+2(-1)^{n-r}, n \geq r . \\
j_{n+r}-j_{n-r}=2^{n-r}\left(2^{2 r}-1\right), n \geq r . \\
j_{n}=J_{n+1}+2 J_{n-1} . \\
j_{n} J_{n}=J_{2 n}, n \geq 0 . \\
J_{m} j_{n}+J_{n} j_{m}=2 J_{m+n} ; \quad J_{m} j_{n}-J_{n} j_{m}=(-1)^{n} 2^{n+1} J_{m-n} .
\end{gathered}
$$

The set of bicomplex numbers is as follows:

$$
B C=\left\{z_{1}+z_{2} \mathbf{j} \mid z_{1}, z_{2} \in C, \mathbf{j}^{2}=-1\right\} .
$$

Here, $\mathbf{i}, \mathbf{j}$ are different and commuting imaginary units, $\mathbf{i j}=\mathbf{j i}, \mathbf{i} \mathbf{i}=\mathbf{j} \mathbf{j}=-1$. Hyperbolic unit $\mathbf{k}$ arises from the multiplication of the two imaginary units $\mathbf{i}$ and $\mathbf{j} ; \mathbf{i j}=\mathbf{k}$. In the set $B C$, the addition operation is component-wise and the multiplication operation is done taking into account the multiplication of the base elements.

Now, let's define a new bicomplex sequence whose coefficients are selected from Jacobsthal Lucas sequence. If we denote $n t h$ bicomplex Jacobsthal number by $j B Q_{n}$, then we can write it as follows: For $n \geq 0$

$$
j B Q_{n}=j_{n}+\mathbf{i} j_{n+1}+\mathbf{j} j_{n+2}+\mathbf{i j} j_{n+3},
$$

where $j_{n}$ is $n t h$ Jacobsthal-Lucas number. Let us write the set of such numbers as follows:

$$
B C_{j}=\left\{j_{C_{n}}+j_{C_{n+2}} \mathbf{j} \mid j_{C_{n}}=j_{n}+\mathbf{i} j_{n+1}, j_{C_{n+2}}=j_{n+2}+\mathbf{i} j_{n+3}, \mathbf{j}^{2}=-1\right\}
$$

Then one can write

$$
B C_{j}=\left\{j B Q_{0}, j B Q_{1}, j B Q_{2}, \ldots, j B Q_{n}, \ldots\right\} .
$$

Notice that the $n t h$ element of this sequence satisfy the following recurrence relation

$$
j B Q_{n}=j B Q_{n-1}+2 j B Q_{n-2} .
$$

For any two elements $j B Q_{n}, j B Q_{m}$ the algebraic operations are as follows:

$$
\begin{gathered}
j B Q_{n}+j B Q_{m}=\left(j_{n}+j_{m}\right)+\left(j_{n+1}+j_{m+1}\right) \mathbf{i}+\left(j_{n+2}+j_{m+2}\right) \mathbf{j}+\left(j_{n+3}+j_{m+3}\right) \mathbf{i j}, \\
j B Q_{n}-j B Q_{m}=\left(j_{n}-j_{m}\right)+\left(j_{n+1}-j_{m+1}\right) \mathbf{i}+\left(j_{n+2}-j_{m+2}\right) \mathbf{j}+\left(j_{n+3}-j_{m+3}\right) \mathbf{i j}, \\
j B Q_{n} j B Q_{m}=\operatorname{sc}\left(j B Q_{n} j B Q_{m}\right)+\operatorname{vec}\left(j B Q_{n} j B Q_{m}\right),
\end{gathered}
$$

where the scalar and vectorial parts are follows.

$$
\begin{gathered}
s c\left(j B Q_{n} j B Q_{m}\right)=\left(j_{n} j_{m}-j_{n+1} j_{m+1}-j_{n+2} j_{m+2}+j_{n+3} j_{m+3}\right) \\
\operatorname{vec}\left(j B Q_{n} j B Q_{m}\right)=\left(j_{n} j_{m+1}+j_{n+1} j_{m}-j_{n+2} j_{m+3}-j_{n+3} j_{m+2}\right) \mathbf{i} \\
+\left(j_{n} j_{m+2}-j_{n+1} j_{m+3}+j_{n+2} j_{m}-j_{n+3} j_{m+1}\right) \mathbf{j} \\
\left.+\left(j_{n} j_{m+3}+j_{n+1} j_{m+2}+j_{n+2} j_{m+1}+j_{(} n+3\right) j_{m}\right) \mathbf{i j}
\end{gathered}
$$

respectively.

\section{Main identities}

In this section we provide some fundamental identities such as the Cassini's, Catalan's, Vajda's identities. The elements of the sequence $\left\{j B Q_{n}\right\}_{n \geq 0}$ can be seen as the coefficients of the power series of the corresponding generating function.

Now, let us give the generating function for the bicomplex Jacobsthal-Lucas numbers.

Theorem 2.1. The generating function for the numbers $j B Q_{n}$ is

$$
G(t)=\frac{j B Q_{0}(1-t)+j B Q_{1} t}{1-t-2 t^{2}}
$$


Proof. Suppose $G(t)$ is a generating function for $j B Q_{n}$

$$
G(t)=\sum_{i=0}^{\infty} j B Q_{i} t^{i}
$$

Multiply this function by $t$ and $t^{2}$, respectively, to take advantage of the recursive relation. Thus, we write

$$
\begin{aligned}
t G(t) & =j B Q_{0} t+j B Q_{1} t^{2}+\ldots+j B Q_{n} t^{n+1}+\ldots \\
t^{2} G(t) & =j B Q_{0} t^{2}+j B Q_{1} t^{3}+\ldots+j B Q_{n} t^{n+2}+\ldots
\end{aligned}
$$

Using the characteristic equation and making needed arrangements, we get

$$
\begin{gathered}
G(t)\left(1-t-2 t^{2}\right)=j B Q_{0}+t\left(j B Q_{1}-j B Q_{0}\right), \\
G(t)=\sum_{i=0}^{\infty} j B Q_{i} t^{i}
\end{gathered}
$$

which is desired result.

Theorem 2.2. For integers $n, r$ such that $n \geq r$, we have

$$
\text { i) } j B Q_{n}+j B Q_{n+1}=3 \underline{\alpha} \alpha^{n} \text {. }
$$

ii) $j B Q_{n+r}-j B Q_{n-r}=\underline{\alpha}\left(2^{n+r}-2^{n-r}\right)$.

Proof. Using the Binet formula the accuracy of the desired equations can be easily seen.

Theorem 2.3. For the bicomplex Jacobsathal-Lucas sequence $j B Q_{n}$, the following equality is then provided:

$$
\sum_{s=1}^{n} j B Q_{s}=\frac{1}{2}\left(j B Q_{n+2}-j B Q_{2}\right)
$$

Proof. In order to prove the claim we will use the following equalities:

$$
\left\{j_{n}\right\}_{n \geq 0}=\{2,1,5,7,17,31,65,127,257,511, \ldots\}
$$

and

$$
\sum_{i=1}^{n} j_{i}=\frac{j_{n+2}-5}{2}
$$

So, we have

$$
\sum_{s=1}^{n} j B Q_{s}=\sum_{s=1}^{n} j_{s}+\mathbf{i} \sum_{s=1}^{n} j_{s+1}+\mathbf{j} \sum_{s=1}^{n} j_{s+2}+\mathbf{i j} \sum_{s=1}^{n} j_{s+3}
$$

This implies that

$$
\sum_{s=1}^{n} j B Q_{s}=\frac{1}{2}\left\{\left(j_{n+2}+\mathbf{i} j_{n+3}+\mathbf{j} j_{n+4}+\mathbf{i j} j_{n+5}\right)-(5+7 \mathbf{i}+17 \mathbf{j}+31 \mathbf{i j})\right\} .
$$

This proves our result. That is,

$$
\sum_{s=1}^{n} j B Q_{s}=\frac{1}{2}\left(j B Q_{n+2}-j B Q_{2}\right) .
$$

The following formula gives any element of the sequence $B C_{j}$ and this formula is known as the Binet formula.

Theorem 2.4. (Binet's Formula) For every positive integer n, the following equality holds.

$$
j B Q_{n}=\underline{\alpha} \alpha^{n}+\underline{\beta} \beta^{n}
$$

where

$$
\underline{\alpha}=(1+2 \boldsymbol{i}+4 \boldsymbol{j}+8 \boldsymbol{i j}) \text { and } \underline{\beta}=(1-\boldsymbol{i}+\boldsymbol{j}-\boldsymbol{i j}) .
$$

Proof. The general term of the sequence $\left\{j B Q_{n}\right\}_{n \geq 0}$ is $j B Q_{n}=A \alpha^{n}+B \beta^{n}$. Using the roots $\alpha, \beta$ of the characteristic equation associated with the recurrence $j B Q_{n}=j B Q_{n-1}+2 j B Q_{n-2}$ we get the values $A$ and $B$ as follows:

$$
A=\frac{j B Q_{1}-j B Q_{0} \beta}{3}, \quad B=\frac{-j B Q_{1}+j B Q_{0} \alpha}{3} .
$$

Writing these values in the equation $j B Q_{n}=A \alpha^{n}+B \beta^{n}$, we have

$$
\begin{gathered}
j B Q_{n}=\frac{j B Q_{1}-j B Q_{0} \beta}{3} \alpha^{n}+\frac{-j B Q_{1}+j B Q_{0} \alpha}{3} \beta^{n}, \\
j B Q_{n}=(1+2 \mathbf{i}+4 \mathbf{j}+8 \mathbf{i j}) \alpha^{n}+(1-\mathbf{i}+\mathbf{j}-\mathbf{i j}) \beta^{n}
\end{gathered}
$$

which is desired. 
Theorem 2.5. For the integers $n, m$ such that $n \geq m$, Catalan's identity is follows:

$$
j B Q_{n+m} j B Q_{n-m}-j B Q_{n}^{2}=\underline{\alpha} \underline{\beta}(\alpha \beta)^{n-m}\left\{(\alpha \beta)^{2 m}-2(\alpha \beta)^{m}+1\right\} .
$$

Proof. From the Binet formula, we write

$$
j B Q_{n+m} j B Q_{n-m}-j B Q_{n}^{2}=\left(\underline{\alpha} \alpha^{n+m}+\underline{\beta} \beta^{n+m}\right)\left(\underline{\alpha} \alpha^{n-m}+\underline{\beta} \beta^{n-m}\right)-\left(\underline{\alpha} \alpha^{n}+\underline{\beta} \beta^{n}\right)^{2} .
$$

If we use the fact $\underline{\alpha} \underline{\beta}=\underline{\beta} \underline{\alpha}$, then we get

$$
\begin{gathered}
j B Q_{n+m} j B Q_{n-m}-j B Q_{n}^{2}=\underline{\alpha} \underline{\beta}\left(\alpha^{n+m} \beta^{n-m}+\alpha^{n-m} \beta^{n+m}-2 \alpha^{n} \beta^{n}\right) . \\
j B Q_{n+m} j B Q_{n-m}-j B Q_{n}^{2}=\underline{\alpha} \underline{\beta}\left(\alpha^{n} \beta^{n}\right)\left\{\frac{\alpha^{m}}{\beta^{m}-1}+\frac{\beta^{m}}{\alpha^{m}-1}\right\} .
\end{gathered}
$$

Hence, after the some calculations we obtain the following equality which is desired result:

$$
j B Q_{n+m} j B Q_{n-m}-j B Q_{n}^{2}=\underline{\alpha} \underline{\beta}(\alpha \beta)^{n-m}\left\{(\alpha \beta)^{2 m}-2(\alpha \beta)^{m}+1\right\} .
$$

Theorem 2.6. For $n \geq 1$, Cassini's identity is follows:

$$
j B Q_{n-1} j B Q_{n+1}-j B Q_{n}^{2}=9 \underline{\alpha} \underline{\beta}(\alpha \beta)^{n-1} .
$$

Proof. One can see that $\underline{\alpha} \underline{\beta}=\underline{\beta} \underline{\alpha}$. Also, by considering the multiplication rules of the base elements we write

$$
j B Q_{n-1} j B Q_{n+1}-\left(j B Q_{n}\right)^{2}=\underline{\alpha} \underline{\beta}\left(\alpha^{n-1} \beta^{n+1}+\alpha^{n+1} \beta^{n-1}-2 \alpha^{n} \beta^{n}\right) .
$$

By making the necessary adjustments and calculations, we obtain

$$
j B Q_{n-1} j B Q_{n+1}-j B Q_{n}^{2}=9 \underline{\alpha} \underline{\beta}(\alpha \beta)^{n-1} .
$$

Thus, we completed the proof.

Notice that Theorem 2.6 is an immediate consequence of Theorem 2.5. In this case, $m=1$, Catalan's formula induces the Cassini's formula:

$$
j B Q_{n-1} j B Q_{n+1}-j B Q_{n}^{2}=9 \underline{\alpha} \underline{\beta}(\alpha \beta)^{n-1} .
$$

Theorem 2.7. (d'Ocagne Identity). For the elements $j B Q_{n}$, we have

$$
j B Q_{m} j B Q_{n+1}-j B Q_{n} j B Q_{m+1}=3 \underline{\alpha} \underline{\beta}\left(\alpha^{n} \beta^{m}-\alpha^{m} \beta^{n}\right) .
$$

Proof. From the Binet's formula, the equality

$$
j B Q_{m} j B Q_{n+1}-j B Q_{n} j B Q_{m+1}
$$

is equal to this:

$$
\underline{\alpha} \underline{\beta}\left\{\alpha^{m} \beta^{n}(\beta-\alpha)+\alpha^{n} \beta^{m}(\alpha-\beta)\right\} .
$$

Hence, we get

$$
j B Q_{m} j B Q_{n+1}-j B Q_{n} j B Q_{m+1}=3 \underline{\alpha} \underline{\beta}\left(\alpha^{n} \beta^{m}-\alpha^{m} \beta^{n}\right) .
$$

\section{Conclusion}

In this study, we introduced the bicomplex number sequence whose coefficients are chosen from the Jacobsthal-Lucas sequence. We gave algebraic properties of the elements of this sequence and obtained their Binet formula. We also obtained the well known some generalized identities related with these numbers such as Cassini and Catalan identities. It should be note that due their applications in different areas, the study of these numbers can give other properties and applications. 


\section{References}

[1] S. Halici, On fibonacci quaternions. Adv. Appl. Clifford Algebr., 22(2) (2012), 321-327.

[2] S. Halici, On Complex Fibonacci Quaternions. Adv. Appl. Clifford Algebr., 23(1) (2013), 105-112.

[3] M. Akyigit, H. H. Kösal, M. Tosun, Fibonacci generalized quaternions, Adv. Appl. Clifford Algebr., 24(3) (2014), 631-641.

[4] J. Cockle, LII. On systems of algebra involving more than one imaginary; and on equations of the fifth degree. The London, Edinburgh, and Dublin Philosophical Magazine and Journal of Science, 35(238) (1849), 434-437.

[5] C. Segre, Le rappresentazioni reali delle forme complesse e gli enti iperalgebrici, Math. Ann., 40 (1892), $413-467$.

[6] G. B. Price, An Introduction to Multicomplex Spaces and Functions, Monographs and Textbooks in Pure and Applied Mathematics, 140, Marcel Dekker, Inc., New York, 1991

[7] G. Dragoni, Sulle funzioni olomorfe di una variable bicomplessa, Reale Acad.d'Italia Mem. Class Sci. Fic. Mat.Nat., 5 (1934),597-665.

[8] M. Futagawa, On the theory of functions of quaternary variable-I, Tohoku Math. J., 29 (1928), 175-222.

[9] A. A. Pogorui, R. M. Rodriguez-Dagnino, On the set of zeros of bicomplex polynomials, Complex Var. Elliptic Equ., 51 7 (2006), $725-730$.

[10] J. Ryan, Complexified Clifford analysis, Complex Var. Elliptic Equ., 1 (1982), 119-149.

[11] S. Halici, On Bicomplex Fibonacci Numbers and Their Generalization, Models and Theories in Soc. Syst., Springer Nature, Studies in Systems, Decision and Control Series, 2019.

[12] M.E. Luna-Elizarraras, E. M. Shapiro, D. C. Struppa, A. Vajiac Bicomplex numbers and their elementary functions, Cubo (Temuco), 14(2) (2012),

[13] Z1- Cerin, Sums of squares and products of Jacobsthal numbers, J. Integer Seq., 10(07.2) (2007), 5.

[14] D. Rochon, M. Shapiro, On algebraic properties of bicomplex and hyperbolic numbers, An. Univ. Oradea Fasc. Mat., 11 (2004),71-110.

[15] S. Halici, Ş. Curuk, On Some Matrix Representations of Bicomplex Numbers, Konuralp Journal of Mathematics, 7(2), (2019), 449-455.

[16] A. F. Horadam, Jacobsthal representation numbers, Significance, 2 (1996), 2-8.

[17] A. F. Horadam, Complex Fibonacci numbers and Fibonacci quaternions, Amer. Math. Monthly, 70(3) (1963), $289-291$.

[18] A. Szynal-Liana, I. Włoch, A note on jacobsthal quaternions, Adv. Appl. Clifford Algebr., 26(1) (2016), 441-447.

[19] D. Savin, Some properties of Fibonacci numbers, Fibonacci octonions, and generalized Fibonacci-Lucas octonions, Adv. Difference Equ., 2015(1) (2015), 298.

[20] T. Koshy, Fibonacci and Lucas Numbers with Applications, Vol 1, John Wiley and Sons, 2001. 